\title{
Functional Aspects and Potential Applications of Weissella Species in Food and Health
}

\author{
Sreya Unnikrishnan and Sreeja V.* \\ Department of Dairy Microbiology, S.M.C. College of Dairy Science, Kamdhenu University, Anand, Gujarat, India \\ *Corresponding author: sreeja.mudgal@kamdhenuuni.edu.in
}

\begin{abstract}
Weissella species are Gram-positive, non-spore-forming, catalase-negative, obligate heterofermentative lactic acid bacteria. They inhabit nutrient rich environments and can be isolated from a variety of sources and different fermented foods. They possess functional and technological attributes which can be of use in the safety, nutritional and sensory characteristics of foods. Among the members of this genus, Weissella cibaria and Weissella confusa have been described as high producers of exopolysaccharides and oligosaccharides, and hence exhibit texturizing properties. Several Weissella species isolated from food and animal sources are reported to possess various health promoting effects like cholesterol reduction, antioxidant activity, immunomodulatory effect, oral health promoting effects and anticancer effects.
\end{abstract}

Keywords: Weissella, Functional, Foods, Health, Lactic acid bacteria

Among the major Genera coming under the Lactic Acid Bacteria (LAB) group, the re-classification of Lactobacillus and Leuconostoc saw emergence of a new Genus called Weissella named after German microbiologist Norbert Weiss. It was proposed as a genus in 1993 (Fessard and Remize, 2017). Members of this genus are Gram-positive, catalase-negative, non-endospore forming cells with coccoid or rodshaped morphology (Abrouel et al. 2015). Species belonging to Weissella are generally non motile in nature with the exception of Weissella behninesis which is motile due to peritrichous flagella (Padonou et al. 2010). Weissella species belong to the phylum Firmicutes, class Bacilli, order Lactobacillales and family Leuconostocaceae (Collins et al. 1993).

Because of the complex nutritional requirements, members of the genus Weissella inhabit (Table 1) nutrient rich environments and can be isolated from a variety of such sources, including vegetables, meat, fish, raw milk, sewage, blood, soil, the gastrointestinal tracts of humans and animals, as well as the oral cavity and uro-genital tract of human (Fusco et al. 2015). Also they are frequently detected in many fermented foods.

Weissella spp. are capable of elaborating bacteriocins, EPS (Exopolysaccharide) and hydrolytic enzymes (Abrouel et al. 2015). Weissella confusa and Weissella cibaria species, are being extensively studied for their ability to produce significant amounts of non-digestible oligosaccharides and extracellular polysaccharides, which can be used as prebiotics or for other applications in food, feed, clinical, and cosmetics industries (Baruah et al. 2019). Members

How to cite this article: Unnikrishnan, S. and Sreeja, V. (2021). Functional Aspects and Potential Applications of Weissella Species in Food and Health. Intl. J. Ferment. Food, 10(1): 13-23.

Source of Support: None; Conflict of Interest: None (क) 
of genus Weisella possess many functional and bio-technological properties, which can enhance nutritional, sensory and bio functional characteristics of foods. Several Weissella strains are reported to possess antimicrobial, antioxidant, anti-inflammatory, chemo-preventive and anti-obesity effects.

Table 1: Habitat of Weissella species

\begin{tabular}{|c|c|}
\hline Species & Habitat or source \\
\hline W. cibaria & $\begin{array}{l}\text { Japanese horseradish, Tomatoes, Orange, } \\
\text { Wheat flour, Corn stovers, Black berries, } \\
\text { Bulgarian traditional Sour dough, Kimchi, } \\
\text { Pickles, Greek Traditional sour dough, } \\
\text { Camel milk, Goat milk, Human faeces \& } \\
\text { Human vagina. }\end{array}$ \\
\hline W. confusa & $\begin{array}{l}\text { Rhizosphere of olive trees, Soil } \\
\text { surrounding rhizosphere, Raw red and } \\
\text { yellow peppers, Heroin, Sugar cane, } \\
\text { Un-pasteurised Brozoa, Masai fermented } \\
\text { milk, Wheat sour dough, Cow milk, } \\
\text { Human faeces \& Human breast milk }\end{array}$ \\
\hline W. halotolerans & $\begin{array}{l}\text { Rhizosphere of olive tree, Soil } \\
\text { surrounding rhizosphere }\end{array}$ \\
\hline W. hellenica & $\begin{array}{l}\text { Vegetative forage crops, Heroin, Raw milk } \\
\text { cheese \& Cow milk }\end{array}$ \\
\hline W. kadleri & Dessert spring, Dessert plants \& Koumiss \\
\hline W. kimchi & $\begin{array}{l}\text { Fluted pumpkin (Telfairia occidentalis) and } \\
\text { green vegetable (Amaranthus spinosus), } \\
\text { Kimchi \& Human vagina }\end{array}$ \\
\hline $\begin{array}{l}W . \\
\text { paramesenteriodes }\end{array}$ & $\begin{array}{l}\text { Fluted pumpkin vegetable (Telfairia } \\
\text { occidentalis) and green vegetable } \\
\text { (Amaranthus spinosus), Semillon and } \\
\text { Sauvignon Blanc grapes, Indian } \\
\text { goosegrass, Mexicanpozol, Goat milk } \\
\text { cheese \& Faeces of breast-fed infants. }\end{array}$ \\
\hline W. soli & Carrots \& Dongchimi \\
\hline
\end{tabular}

Sourced from Fusco et al. 2015.

\section{History and Taxonomy}

Collins and colleagues were the first to designate the genus Weissella in 1993 after taxonomic studies on atypical Leuconostoc-like microorganisms which came from fermented sausages produced in Greece. Further molecular systematic investigations suggested that Leuconostocs could be separated into three distinct genetic lineages. Study based on phenotypic, biochemical and 16S rRNA gene analyses allowed the differentiation of the new genus Weissella and reassignment of the species previously grouped in the genus Lactobacillus as W. confusa, W. halotolerans, $W$. kandleri, $W$. minor, and $W$. viridescens (Abriouel et al. 2015).

\section{Biochemical Characteristics}

Weissella bacteria are facultatively anaerobic chemoorganotrophs with an obligately fermentative metabolism. They do not possess cytochromes and ferment glucose heterofermentatively via the hexosemonophosphate and phosphoketolase pathways. End products of glucose heterofermentation include lactic acid (with some species producing only $\mathrm{D}(-)$ and others both $\mathrm{D}(-)$ and $\mathrm{L}(+)$ lactic acid enantiomers), gas $\left(\mathrm{CO}_{2}\right)$ and ethanol and/or acetate (Bjrkroth et al. 2014). Biochemical and sugar fermentation characteristics of different Weissella species are shown in Table 2 and Table 3.

\section{Molecular Characteristics}

DNA: DNA hybridization analyses, together with phenotypic data in a polyphasic taxonomical approach grouped Weissella in five phylogenetic branches based on $16 \mathrm{~S}$ phylogeny. The genome sequence of 28 strains belonging to 12 species has been published. The smallest genomes belonged to Weissella halotolerans $(1.36 \mathrm{Mb})$ and W. ceti $(1.35$ to 1.39 $\mathrm{Mb}$ ), which share the same taxonomic branch. The largest genomes belonged to $W$. cibaria, W. confusa, $W$.jogaejeotgali and W. oryzae. The core-proteome of Weissella represented 729 COGs (Clusters of Orthologous Genes), over a pan-proteome of 4712 COGs (Abriouel et al. 2015).

\section{Bacteriocins elaborated by Weissella spp.}

Several Weissella species produce bacteriocins (Table 4) and shows broad spectrum antimicrobial activity against different food borne pathogens. The first bacteriocin produced by Weissella strains to be discovered was Weissellicin 110 in the year 2007. This bacteriocin has antimicrobial activity against some 
Table 2: Biochemical characteristics of Different Weissella species

\begin{tabular}{lllllll}
\hline Characteristics & W. cibaria & W. confusa & W. paramesentriods & W. hellenica & W. koreensis & W. halotolerence \\
Esculin hydrolysis & + & + & V & ND & - & - \\
Ammonia from Araginine & + & + & - & - & - & + \\
Dextran formation & + & + & - & D & D & DL \\
Lactic acid configuration & DL & DL & D & $39-40$ & 37 & 44 \\
Mol \% G+C content & $44-45$ & $45-47$ & 40.6 & & + \\
\hline
\end{tabular}

(+ $90 \%$ or more strains are positive), (- 90 or more strains are negative (d-11-89\% of strains are positive, (ND-No data available), (V-Variable) Sourced from Fusco et al. (2015).

Table 3: Sugar fermentation characteristics of Weissella species

\begin{tabular}{|c|c|c|c|c|c|c|}
\hline Acid Produced from & W. cibaria & W. confusa & W. paramesenteroides & W. hellenica & W. koreensis & W. halotolerans \\
\hline Arabinose & + & - & $\mathrm{D}$ & + & + & - \\
\hline Fructose & + & + & + & + & ND & + \\
\hline Galactose & - & + & + & - & - & - \\
\hline Maltose & + & + & + & + & - & + \\
\hline Raffinose & - & - & $\mathrm{D}$ & - & - & - \\
\hline Ribose & - & + & $\mathrm{D}$ & + & + & + \\
\hline Sucrose & + & + & + & - & - & - \\
\hline Xylose & + & + & $\mathrm{D}$ & - & + & - \\
\hline
\end{tabular}

(+ $90 \%$ or more strains are positive), (- 90 or more strains are negative, (d-11-89\% of strains are positive, (ND-No data available) Fusco et al. 2015.

Table 4: Bacteriocins produced by Weissella species

\begin{tabular}{llll}
\hline Name of Bacteriocin & Producing organism & Class & Reference \\
\hline Weissellicin 110 & W. cibaria 110 & Unclassified & (Srionnual et al. 2007) \\
Weissellin A & W. parameseteriods Dx & Class IIA & (Papggianni et al. 2011) \\
Weissellicin L & W. hellenica 4-7 & Unclassified & (Leong et al. 2013) \\
Weissellicin D & W. hellenica D1501 & Unclassified & (Chen et al. 2014) \\
Weissellicin M & W. hellenica QU13 & Unclassified & (Masuda et al. 2012) \\
Weissellicin Y & W. hellenica QU13 & Unclassified & (Masuda et al. 2012) \\
\hline
\end{tabular}

Gram-positive microorganisms and it is resistant to high temperatures and catalase (Srionnual et al. 2007). The listericidal bacteriocin weissellin A was further investigated for its technological application in fermented sausages (Fusco et al. 2017). Bacteriocinogenic strain $W$. hellenica D1501 was successfully used to enhance the shelf-life of tofu (Chen et al. 2014). Weissellicin MBF is encoded by a large transferable plasmid of $17.6 \mathrm{kbp}$, pWcMBF8-1.
It was the first study which reported plasmidassociated bacteriocin activity in the genus Weissella (Malik et al. 2016).

\section{FUNCTIONAL ASPECTS}

\section{Antimicrobial activity}

Several Weissella strains produce a variety of antagonistic substances, including organic acids and 
bacteriocins. Weissella cibaria TM128 strain showed inhibition against phyto-pathogens Monilinia laxa, Erwinia carotovora and Xanthomonas campestris by production of organic acids and hydrogen peroxide (Trias et al., 2008). Singh et al. (2015) explored bacterial strain, Weissella oryzae DC6, isolated from mountain ginseng, for the synthesis of silver nanoparticles. The synthesized nanoparticles were evaluated for their antimicrobial activity against clinical pathogens including Vibrio parahaemolyticus, Bacillus cereus, Bacillus anthracis, Staphylococcus aureus, Escherichia coli, and Candida albicans. Further the potential of nanoparticles has been observed for biofilm inhibition against Staphylococcus aureus and Pseudomonas aeruginosa. Their study concluded that the synthesis of silver nanoparticles by the strain $W$. oryzae DC6 may serve as a simple, green, cost-effective, consistent, and harmless method to produce antimicrobial silver nanoparticles. W. confusa K75KT361205 strain isolated from vaginal swab showed highest inhibition against $P$. aeruginosa (MTCC3541) followed by Proteus mirabilis (MTCC428).The results indicated that this particular strain can be used for treating vaginal infections (Purkhayastha et al. 2017).

Yu et al. (2019) reported that antimicrobial activity of W. cibaria JW15 is stronger than probiotic strain Lactobacillus rhamnosus GG (LGG). Weissella strain showed higher inhibition against Listeria monocytogenes and Salmonella enteritidis and also showed higher lactic acid and acetic acid production than LGG (Yu et al. 2017). Huy et al. (2020) reported that W. cibaria HN05 isolated from white pacific shrimp exhibited strongest antagonistic activity against Vibrio parahaeamolyticus with antagonistic activity 220 $\mathrm{AU} / \mathrm{ml}$ to $500 \mathrm{AU} / \mathrm{ml}$. W. cibaria HN05 inhibited E. coli growth with antagonistic activity of $240 \mathrm{AU} / \mathrm{ml}$. Fhoula et al. (2018) reported that W. halotolerans F99 isolated from camel faeces showed highest zone of inhibition against $P$. aeruginosa ATCC27853 $(24.6 \mathrm{~mm})$ followed by $S$. typhimurium IPT13 (21.4 mm).

\section{Antioxidant activity}

The cell free extracts (CFS) and EPS from several Weissella strains isolated from fermented foods shows high antioxidant activity. They produce several antioxidantenzymesincluding superoxidedismutase, Glutathione reductase and NADH oxidase. Sharma et al. (2018) studied the anti-oxidative activity of $W$. confusa KR780676, isolated from an Indian traditional fermented food (Idli batter). Cell-free extract showed prominent hydroxyl radical scavenging activity, while intact cells exhibited significant DPPH, superoxide anion radical scavenging potential and lipid peroxidation inhibition. W. cibaria GA44 isolated from Gari showed antioxidant activity. The in vitro antioxidant activities of the EPS showed good scavenging effects on superoxide anion radical and hydroxyl radical (Dahnusi et al. 2018). Yu et al. (2019) studied the antioxidant property of W. cibaria JW15. They have found that intact JW15 cells showed radical scavenging effect ranging from 23.53 to $60.85 \%$ and inhibited lipid peroxidation of $33.31 \%$ in the tested assays. Additionally, JW15 exhibited a statistical difference on the antioxidant effect compared to $L$. rhamnosus GG.

Amrutha et al. (2019) studied the anti-oxidant activity of Weissella strains isolated from tender coconut water. They have found that the EPS produced by W. cibaria DMA 18 displayed remarkable antioxidant activity. DPPH assay revealed antioxidant activity of $27 \%$ and $33.55 \%$ respectively with sample volumes as low as $10 \mu \mathrm{l}$ and $6 \mu \mathrm{l}$ respectively. The results confirm the potential of $W$. cibaria to be used as a functional culture for designing foods to ameliorate disorders induced by oxidative stress. Mercha et al. (2020) reported that Weissella strains isolated from camel milk showed higher anti-oxidant activity. $W$. confusa strain showed 59.01 to $63.31 \%$ and for W. cibaria 52.68 to $62.41 \%$ free radical scavenging activity was recorded. The $W$. confusa I17 recorded the highest scavenging ability (63.31\%).

\section{Exopolysaccharide production}

EPS produced by strains of the genus Weissella (Table 5) are homopolysaccharides (HoPS) such as dextran, fructan, galactan, levan and inulin. Strains of the genus Weissella use sucrose as the obligatory substrate for the extracellular synthesis of HoPS. The enzymes 
Table 5: EPS Produced by different Weissella strains

\begin{tabular}{llll}
\hline Species & Substrate & EPS & Reference \\
\hline W. cibaria RBA12 & Sour dough & Dextran & (Baruah et al. 2016) \\
W. confuse & Rye bran & Dextran & (Kajala et al. 2016) \\
W. confusa KR780676 & 2\% sucrose supplemented MRS agar & Linear exopolysaccharide, Galactan & (Devi et al. 2016) \\
W. confusa $\&$ W. cibaria & MRS agar supplemented 10\% & Dextran, Fructan, Leavan, Inulin & Malang (et al. 2015) \\
& sucrose & &
\end{tabular}

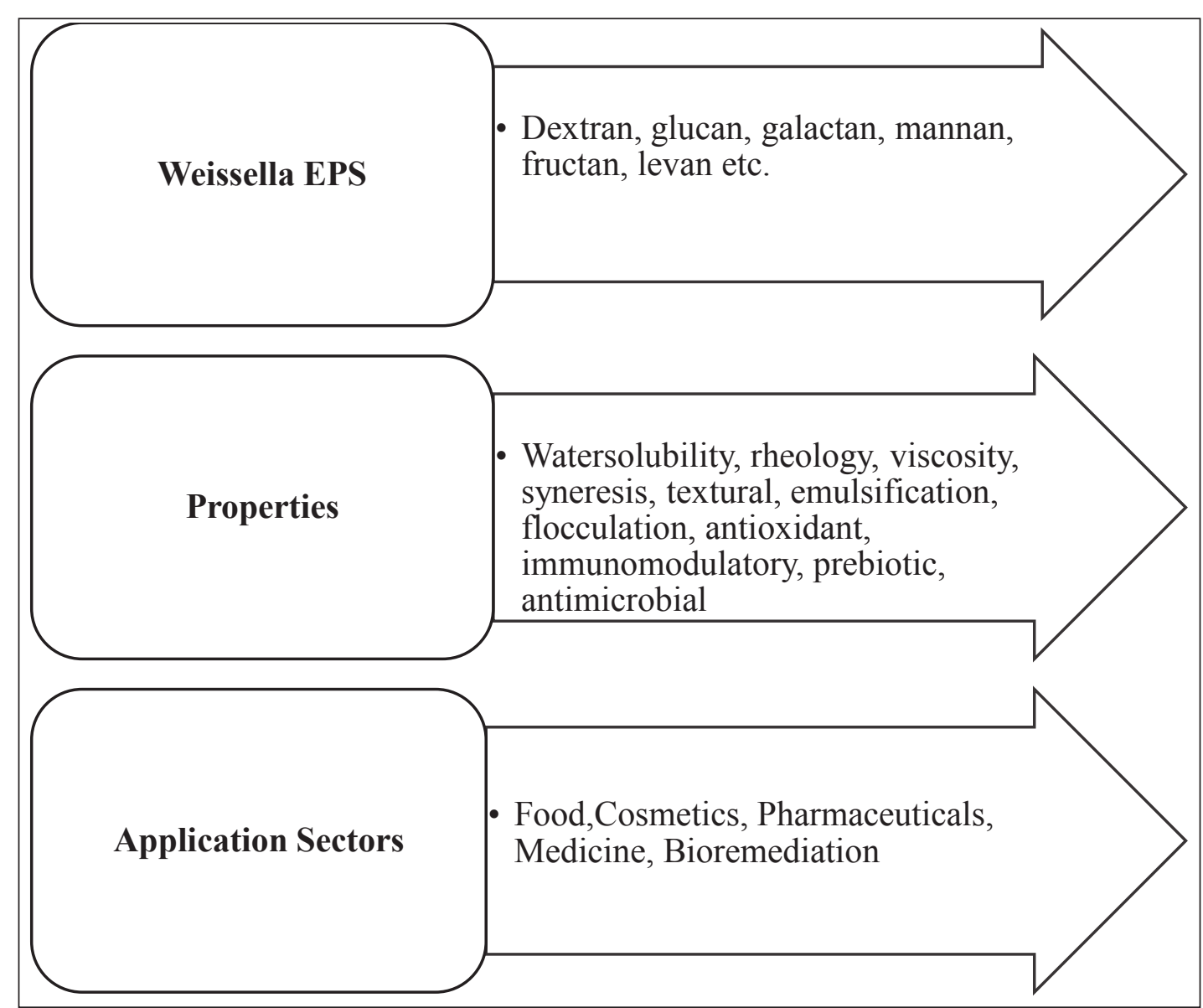

Fig. 1: Types of EPS, Properties and Application Sector (Sourced from Baruah et al., 2019)

used to hydrolyze sucrose are glycosyltransferase, glucan-sucrase (GS) or fructan-sucrase (FS) (Teixeira et al. 2018). W. confusa and W. cibaria have ability to produce significant amounts of dextran. Dextran produced by Weissella spp. have similar structures with mainly $\alpha-(1-6)$ linkages and only $\alpha-(1-3)$ linkages (Malang et al. 2016). The EPS produced by Weissella species possess different properties like water solubility, rheology, viscosity, textural, emulsification, flocculation, antioxidant, immunomodulatory, prebiotic, antimicrobial properties (Fig. 1).

Galle et al. (2011) screened EPS producing Weissella strains for use as starter in sorghum and wheat sourdough. W. cibaria MG1 produced considerable amount of dextran (0.6 to $8.0 \mathrm{~g} / \mathrm{kg}$ sourdough) which 
were supplemented with $15 \%$ sucrose. Incorporation of dextran enriched sourdough $(10 \%$ and $20 \%)$ provided mildly acidic wheat bread with improved bread quality.

\section{APPLICATION IN FOOD INDUSTRY}

\section{In Bread baking}

W. cibaria MG1 was reported as a suitable starter culture for sourdough fermentation of buckwheat, quinoa and teff flour (Wolter, 2012). Galle et al. (2011) screened the EPS-forming Weissella strains for their potential use as starter strains in sorghum and wheat sourdoughs strains $W$. kimchii and W. cibaria MG1 produced dextrans in concentrations high enough to be used as potential replacers of non-bacteria hydrocolloids, such as guar gum and Hydroxyl poly methyl cellulose (HPMC) in gluten-free sourdoughs bread. Malang et al. (2015). reported that the EPS dextran, levan and ropy capsular polysaccharide, produced by $W$. confusa were evaluated in breads and the delaying of the deterioration by fungus, as well as improving of the texture. Kajala et al. (2015) characterized, and cloned the potential of dextransucrase obtained from $W$. confusa VTT E-90392 in preparing bran for use as a baking aid in high fiber baking. They have concluded that presence of dextran improved bread softness and neutralized bran-induced volume loss.

\section{As Texturizing Agent}

Use of EPS dextran produced by W. cibaria TN610 to improve textural properties of semi-skimmed milk supplemented with sucrose was reported by Bejar et al. (2013). This strain has potential in the application as a safe additive in food to improve the texture of dairy products. W. cibaria MG1 is capable of producing dextran \& glucooligosaccharides. During sucrose-supplemented barley-malt-derived wort fermentation up to $36.4 \mathrm{~g} / \mathrm{l}$ of dextran was produced in an optimized system, which improved the rheological profile of the resulting fermentate (Zanini et al. 2013). Quinoa-based yoghurt fermented with dextran producer $W$. cibaria MG1 was developed by
Zanini et al. (2015). Concentration of EPS (40 mg/L) guaranteed the high water retention capacity and viscosity ( $0.57 \mathrm{mPa}$ s) of the final product. Dextran from $W$. confusa was able to improve the texture and sensory properties of pureed carrots with pleasant odor and flavor (Juvonen et al. 2015).

\section{As an adjunct culture in Cheddar cheese making}

Lynch et al. (2013) examined the potential of dextran producing Weissella MG1 for use in Cheddar cheese manufacture as an adjunct starter. The strain survived in cheese with levels increased by $1.5 \log$ cycles over the ripening period. It also increased the moisture retention of cheese without significantly affecting cheese proteolysis.

\section{Application in food packaging}

Woraprayote et al. (2018) developed a biodegradable food packaging with antimicrobial properties by incorporating the Bacteriocin 7293 produced by the strain W. hellenica BCC 7293. They reported that use of this controlled pathogenic bacteria in fillets of pangasius fish. The film produced inhibited the multiplication of both Gram-positive bacteria such as L. monocytogenes and S. aureus as well as Gramnegative bacteria such as $P$. aeruginosa, Aeromonas hydrophila, E. coli and Salmonella typhimurium

\section{Vitamin production}

Some strains of $W$. cibaria and $W$. confusa also have the capacity to produce folate (vitamin B9), which allows the nutritional improvement of fermented products that use these strains in the fermentation process. Divya et al. (2012) studied the folate production of Weissella strains. W cibaria strain isolated from pickle produced $11.2 \mathrm{ng} / \mathrm{ml}$ of folate in the medium. They have concluded that further development of the bioprocess using the strain $W$. cibaria can enhance the bioavailability of this vitamin.

The use of glutaminase producing W. cibaria MSS2 as starter culture for the production of Nham fermented sausage and for kimchi was reported by Abriouel et al. (2015). 


\section{HEALTH APPLICATIONS}

\section{Probiotic Potential}

Aiming at developing novel probiotic foods or probiotic animal feeds, many researchers have isolated and screened Weissella strains from humans. However, only few studies investigated the probiotic potential of Weissella strains using in vivo studies. Patel et al. (2012) reported that W. confusa AI10 was the most resistant strain to bile salts $(0.3 \%)$, with $72 \%$ of survival after $24 \mathrm{~h}$ at $37{ }^{\circ} \mathrm{C}$. On the other hand, $L b$. plantarum AD29 was the less resistant, with $14 \%$ of survival. In the same study, W. cibaria 142 showed $31 \%$ of survival after $2.5 \mathrm{~h}$ at $37{ }^{\circ} \mathrm{C}$ in MRS broth adjusted to $\mathrm{pH} 3$, which demonstrated the ability of this strain to grow in acidic conditions. Anandharaj et al. (2015) reported that W. koreensis FKI21 was the best resistant strain to $\mathrm{pH} 1.0$, with $29.8 \%$ of survival, while $L b$. crispatus GI6 showed lower resistance with $18.3 \%$ of survival. In this same study, W. koreensis and $L b$. crispatus strains showed approximately the same resistance profile to $0.3 \%$ and $0.5 \%$ of bile salts.

Elavarsi et al. (2015) reported that W. cibaria KTSMBNL 28 isolated from goat milk exhibited high potential probiotic properties. This strain was able to tolerate $\mathrm{pH} 3.0$ maintained in gastric fluid and resist upto $1 \%$ of bile salt retained in intestinal fluid. Moreover, KTSMBNL28 recorded highest level of hydrophobicity (35-70\%) and its non-hemolytic property ensured it's safety.

Wang et al. (2020) isolated W. confusa from human fecal samples and evaluated its probiotic properties. Similar to probiotics, W. confusa could inhibit pathogen growth and could tolerate high bile salt concentration and low $\mathrm{pH}$. The survival rate in $0.3 \%$ concentration bile salt of $W$. confusa strains isolated from human ranged from 2.2 to $128.8 \%$, and its acid tolerance ranged from 2.4 to $20.2 \%$. Mercha et al. (2020) reported that $W$. confusa, W.cibaria isolated from camel milk showed great probiotic potential. These isolates were resistant to simulated gastrointestinal tract conditions, presented noticeable hydrophobicity and auot-aggregation percentages, high EPS and diacetyl production abilities, as well as significant acidifying, proteolysis and autolysis activities. These isolates also exhibited antibacterial activity against a wide range of pathogenic bacteria and did not present hemolytic and DNase activities.

\section{Oral health}

W. cibaria strain CMU (Chonnam Medical University) has shown oral colonizing ability and inhibitory effects against the formation of volatile sulphur compounds (VSC). Weissella cibaria strains showed inhibitory effects against biofilm formation.

W. cibaria isolates from children's saliva were shown to inhibit in vitro biofilm formation and proliferation of $S$. mutans, causative agent of early childhood dental caries, by production of polymers from sucrose (Kang et al. 2005). In an In vivo study on 72 volunteers who rinsed their teeth after brushing in the morning, afternoon and evening, with a rinse that contained the probiotic $W$. cibaria CMU significantly reduced plaque index by $20 \%$ (Kang et al. 2005). Gargling with oral probiotic solution containg W. cibaria CMS1 and CMS2 was resulted in reduction in hydrogen sulphide (H2S) $(48.2 \%)$ and methanethiol $\left(\mathrm{CH}_{3} \mathrm{SH}\right)$ $(59.2 \%)$ and inhibition of Fusobacterium nucleatum by production of hydrogen peroxide was observed (Kang et al. 2006).

Jang et al. (2016) conducted a study to compare the characteristics of oral care probiotics. W. cibaria CMU and 4 commercial probiotic strains were used in the study. W. cibaria produced less acid and more hydrogen peroxide than other four strains during the study. They have also observed that W. cibaria inhibited the biofilm formation by Streptococcus mutans (97\% inhibition) at lower level concentration $\left(5 \times 10^{8}\right.$ cells $\left./ \mathrm{ml}\right)$. They have concluded that $W$. cibaria CMU can be used as an oral care probiotic.

Park et al. (2018) studied the effect of diet containing $W$. cibaria CMU on $S$. mutans causing dental caries. The participants were divided randomly in to two groups and they have been instructed to take the tablets daily. The results revealed that $W$. cibaria $\mathrm{CMU}$ diet significantly supressed the biofilm formation on the surface enamel. Oral colonization of $W$. cibaria 
was also observed which prevented the proliferation of S. mutans and prevented the formation of dental caries.

Lim et al. (2018) reported that the cell free supernatant (CFS) from W. cibaria CMU showed inhibitory effect on $F$. nucleatum. They have identified several antimicrobial compounds like hydrogen peroxide, organic acids and free fatty acids. The CFS was also detected for the secretory protein $\mathrm{N}$-actyalmuramidase having antimicrobial activity against Prophyromonas hydrophilla.

Kim et al. (2019) reported that CFS of W. cibaria CMU (OraCMU) suppressed the production of volatile sulphur compounds not only through its bactericidal effects on $P$. gingivalis, but also via its inactivating effects on bacterial METase. Do et al. (2019) conducted a study to analyse the effects of $W$. cibaria $\mathrm{CMU}$ on canine oral health. There was a significant reduction in methyl mercaptan, plaque index in the CMU treated groups, and also these groups showed significant decrease in F. nucleatum, Prophromonas gingivalis, Prevotella intermedia and Tannerella forsythia. They have concluded that W. cibaria have the ability to colonize in the canine oral cavity and it supressed the halitosis and inhibited the proliferation of mal odour causing oral bacteria in beagles.

Kang et al. (2019) evaluated the safety of W. cibaria CMU and CMSI through genotypic and phenotypic analysis. These strains have been registered as safe raw materials by the Korea Food and Drug Administration (KFDA) and are actively commercialized as oral care probiotics in Korea. Both strains tested negative for haemolytic activity, mucin degradation, platelet aggregation and toxin production. They have observed that there was no antibiotic gene transferability and virulence gene presence in both strains.

Kang et al. (2020) reported that oral administration of $W$. cibaria CMU (OraCMU) containing tablets can improve periodontal health and oral microbiota. They have conducted a trial on double blind, placebocontrolled groups in 92 adults without periodontitis. The tablets were administrated once daily basis for 8 weeks. They have observed periodontal clinical parameters including bleeding on probing (BOP), probing dept (PD), gingival index (GI) and plaque index They have found that BOP improved more in probiotic group over 8 weeks.

\section{Anticancer activity}

Cha et al. (2008) studied the anticancer activity of $W$. cibaria in colorectal cancer. W. cibaria was incubated for 24 hours in MRS (Deman Rogosa Sharpe) badge, diluted with phosphate-buffered saline, and 10\% concentration of bacteria samples were provided to normal cell strains and colorectal cancer cells strains for 72 hours. After incubation, the suspension of cell growth was measured using the MTT assay. Cell growth was suppressed by treatment of $W$. cibaria in colorectal cancer cells but not in normal cells.

\section{Cholesterol reduction}

Use of $W$. cibaria as a starter for food fermentation promoted the formation of ornithine from arginine, which in turn provide health beneficial effects, such as anti-obesity effects due to high levels of ornithine in fermented food (Kwak et al. 2014). Kim et al. (2006) reported that W. kimchi KCTC3746 and W. confusa KCTC 3499 showed cholesterol lowering effect of about 55\%. Moon et al. (2012) in an in vitro study demonstrated that intracellular lipid accumulation in 3T3-L1 cells could be inhibited by the ornithine rich cytoplasmic extract of $W$. koreensis OK1-6. Also, cytoplasmic fraction of $W$. koreensis OK1-6 reduced the expression level of lipogenic gene resulting in the reduced triglyceride level in treated 3T3-L1 cells compared with control groups. W. halotolerans F99 isolated from camel faeces was found to reduce cholesterol in vitro by $49 \%$ also the same strain was evaluated for the serum lipid metabolism in Wistar rats fed with a high cholesterol diet. Study results revealed that compared with rats fed a high-fat (HF) diet without Weissella administration, total serum cholesterol, low-density lipoprotein cholesterol, and triglycerides levels were significantly reduced in $W$. halotolerans F99-treated HF rats, with no significant change in high-density lipoprotein cholesterol 
HDL-C levels. W. koreensis FK121 isolated from traditionally fermented Koozh was able to assimilate more cholesterol from growth medium than other tested strain. Cholesterol assimilation $(\mu \mathrm{g} / \mathrm{ml})$ for $W$. koreensis FK121 was about $56.25 \%$ with bile salts.

\section{Anti-inflammatory and immunomodulatory activity}

Ahn et al. (2013) reported that the immune control effect of $W$. cibaria was stronger than the well-known probiotic bacterium, L. rhammosus GG (LGG). W. cibaria produced higher levels of nitric oxide, nuclear factor (NF)- $\kappa \mathrm{B}$, cytokines (e.g., interleukin-1 $\beta$ and tumor necrosis factor- $\alpha$ ) than LGG, suggesting that $W$. cibaria is more effective in immune control compared to LGG. Lim et al. (2018) applied W. cibaria WIKIM28 isolated from gatkimchi in a mouse atopic dermatitis (AD) model and found that this strain can ameliorate AD-like symptoms by suppressing allergic Th2 responses. These results suggest a potential application of W. cibaria WIKIM28 as a dietary supplement or a therapeutic agent to ameliorate AD. Lee et al. (2018) investigated the impact of consuming W. cibaria JW15 on natural killer (NK) cell activity, cytokines and immunoglobulins (Igs) in 100 nondiabetic participants. Administration of W. cibaria JW15 for 8 weeks improved the overall immunity in probiotic group by stimulating the natural killer cells activity. Administration of W. cibaria JWI5 in an immunosuppressed mouse by cyclophosphamide increased the number of WBC and splenocyte cells and stimulated the production of cytokines (TNF- $\alpha$ and IL-6) (Park et al. 2018).

Weissella strains isolated from idli batter and infant faeces were tested for cholesterol reduction, adhesion to Caco-2 cells and mucin and their ability to prevent LPS-induced nitric oxide and proinflammatory cytokines. All the strains suppressed the production of TNF $\alpha$ (Tumor necrosis factor alpha), IL-6 (Interleukin-6) and IL-8 (Interleukin-8) cytokines in murine macrophage model and suppressed the production of IL-8 in Caco-2 cell model (Singh et al. 2018). Yu et al. (2019) evaluated the anti-inflammatory potential of W. cibaria JW15 against lipopolysaccharide (LPS) stimulation. Heat- killed JW15 displayed anti-inflammatory potential by alleviating the pro-inflammatory features in LPSinduced RAW 264.7 cells through suppressing NF$\kappa B$ activation. The cellular signaling pathways of its anti-inflammatory effect were involved in inhibition of MAPKs activation.

\section{SAFETY ASPECTS}

\section{Clinical Infections Associated with Weissella Species}

Weissella strains have been isolated from clinical specimens such as blood, skin, infected wounds and feces of both humans and animals. W. cibaria, the only species of Weissella, which have been described as opportunistic pathogens of humans or as emerging pathogen for farmed rainbow trouts. W. ceti has been recognized as etiological agent of weissellosis, an emergent disease occurring in farmed rainbow trout (Oncorhynchus mykiss) which cause septicemia with a high mortality rate (Fusco et al. 2015).

The presence of several virulence determinants such as collagen adhesins, aggregation substances, hemolysin, mucus binding proteins and staphylococcal surface protein are reported in strains of $W$. ceti, W. cibaria, W. confusa, W. halotolerans, W. hellenica, W. koreensis, W. oryzae, W. paramesenteroides and W. thailandensis (Abriouel et al. 2015).

\section{CONCLUSION}

Weissella is now a well delineated genus in the lactic acid bacteria and the members of this genus occur in a variety of habitats. Weissella species produce functional biomolecules such as bacteriocins, EPS and hydrolytic enzymes having potential applications in food and health. W. cibaria strains are well known for oral health applications. However, the safety aspects of Weissella should be taken into consideration prior to its application in the food and health industry.

\section{REFERENCES}

Abriouel, H., Lerma, L.L., Casado Muñoz, M.D.C., Montoro, B.P., Kabisch, J., Pichner, R. and Gálvez, A. 2015. The controversial nature of the Weissella genus: technological and functional aspects versus whole-genome analysis- 
based pathogenic potential for their application in food and health. Frontiers in Microbiology, 6: 1197.

Adesulu-Dahunsi, A.T., Sanni, A.I. and Jeyaram, K. 2018. Production, characterization and In vitro antioxidant activities of exopolysaccharide from Weissella cibaria GA44. LWT: 87: 432

Ahn, S.B., Park, H.E., Lee, S.M., Kim, S.Y., Shon, M.Y. and Lee, W.K. 2013. Characteristics and immuno-modulatory effects of Weissella cibaria JW15 isolated from Kimchi, Korea traditional fermented food, for probiotic use. Journal of Biomed Reserch, 14(206): 11.442.

Anandharaj, M., Sivasankari, B., Santhanakaruppu, R., Manimaran, M., Rani, R.P. and Sivakumar, S. 2015. Determining the probiotic potential of cholesterol-reducing Lactobacillus and Weissella strains isolated from gherkins (fermented cucumber) and south Indian fermented koozh. Research in Microbiology, 166(5): 428-439.

Baruah, R., Maina, N.H., Katina, K., Juvonen, R. and Goyal, A. 2017. Functional food applications of dextran from Weissella cibaria RBA12 from pummelo (Citrus maxima). International Journal of Food Microbiology, 242: 124-131.

Bejar, W., Gabriel, V., Amari, M., Morel, S., Mezghani, M., Maguin, E., ... and Chouayekh, H. 2013. Characterization of glucansucrase and dextran from Weissella sp. TN610 with potential as safe food additives. International Journal of Biological Macromolecules, 52: 125-132.

Elavarasi, V., Pugazhendhi, A., Poornima Priyadharsani, T. K., Valsala, H. and Thamaraiselvi, K. 2014. Screening and characterization of Weissella cibaria isolated from food source for probiotic properties. International Journal of Computer Applications, 1: 29-32.

Fessard, A. and Remize, F. 2017. Why are Weissella spp. not used as commercial starter cultures for food fermentation. Fermentation, 3(3): 38.

Fusco, V., Quero, G.M., Cho, G.S., Kabisch, J., Meske, D., Neve, H. and Franz, C.M. 2015. The genus Weissella: taxonomy, ecology and biotechnological potential. Frontiers in Microbiology, 6: 155.

Fhoula, I., Rehaiem, A., Najjari, A., Usai, D., Boudabous, A., Sechi, L.A. and Hadda-Imene, O. 2018. Functional Probiotic Assessment and In Vivo Cholesterol-lowering Efficacy of Weissella sp. Associated with Arid Lands Living Hosts. Biomed Research International, 1(7): 11.

Galle, S., Schwab, C., Arendt, E. and Gänzle, M. 2010. Exopolysaccharide-forming Weissella strains as starter cultures for sorghum and wheat sourdoughs. Journal of Agricultural and Food Chemistry, 58(9): 5834-5841.

Lee, Y.J., Lee, A., Yoo, H.J., Kim, M., Noh, G.M. and Lee, J.H. 2018. Supplementation with the probiotic strain Weissella cibaria JW15 enhances natural killer cell activity in nondiabetic subjects. Journal of Functional Foods, 48: 153158.
Huy, N.D., Ngoc, L.M.T., Loc, N.H., Lan, T.T. and Quang, H.T. 2020. Isolation of Weissella cibaria from Pacific white shrimp (Litopenaeus vannamei) gastrointestinal tract and evaluation of its pathogenic bacterial inhibition. Indian Journal of Science \& Technology, 13(10): 1200-1212.

Kajala, I., Shi, Q., Nyyssölä, A., Maina, N.H., Hou, Y., Katina, K., ... and Juvonen, R. 2015. Cloning and characterization of a Weissella confusa dextransucrase and its application in high fibre baking. Public Library of Science, 10(1): 116418.

Kang, M.S., Kim, B.G., Chung, J., Lee, H.C. and Oh, J.S. 2006. Inhibitory effect of Weissella cibaria isolates on the production of volatile sulphur compounds. Journal of Clinical Periodontology, 33(3): 226-232.

Kang, M.S., Chung, J., Kim, S.M., Yang, K.H. and Oh, J.S. 2006. Effect of Weissella cibaria isolates on the formation of Streptococcus mutans biofilm. Caries Research, 40(5): 418-25.

Kang, M.S., Yeu, J.E. and Hong, S.P. 2019. Safety Evaluation of oral care probiotics Weissella cibaria CMU and CMS1 by phenotypic and genotypic analysis. International Journal of Molecular Sciences, 20(11): 2693.

Kang, M.S., Lee, D.S., Lee, S.A., Kim, M.S. and Nam, S.H. 2020. Effects of probiotic bacterium Weissella cibaria CMU on periodontal health and microbiota: a randomised, doubleblind, placebo-controlled trial. BMC Oral Health, 20(1): 1-12.

Kwak, S.H., Cho, Y.M., Noh, G.M. and Om, A.S. 2014. Cancer preventive potential of kimchi lactic acid bacteria (Weissella cibaria, Lactobacillus plantarum). Journal of Cancer Prevention, 19(4): 253-258.

Lim, H.S., Yeu, J.E., Hong, S.P. and Kang, M.S. 2018. Characterization of antibacterial cell-free supernatant from oral care probiotic Weissella cibaria, CMU. Molecules, 23(8): 1984.

Malik, A., Sumayyah, S., Yeh, C.W. and Heng, N.C. 2016. Identification and sequence analysis of pWcMBF8-1, a bacteriocin-encoding plasmid from the lactic acid bacterium Weissella confusa. FEMS Microbiology Letters, 363(8).

Moon, Y.J., Soh, J.R., Yu, J.J., Sohn, H.S., Cha, Y.S. and Oh, S.H. 2012. Intracellular lipid accumulation inhibitory effect of Weissella koreensis OK 1-6 isolated from Kimchi on differentiating adipocyte. Journal of Applied Microbiology, 113(3): 652-658.

Park, H.E. and Lee, W.K. 2018. Immune enhancing effects of Weissella cibaria JW15 on BALB/c mice immunosuppressed by cyclophosphamide. Journal of Functional Foods, 49: 518525.

Purkayastha, S.D., Bhattacharya, M.K., Prasad, H.K., Upadhyaya, H., Lala, S.D., Pal, K. and Sharma, G.D. 2017. Antibacterial activity of Weissella confusa isolated from vaginal swab of Indian women. International Journal of Advances in Chemical Engineering \& Biological Science, 4(1): 98-102. 
Singh, P., Kim, Y.J., Wang, C., Mathiyalagan, R. and Yang, D.C. (2016). Weissella oryzae DC6-facilitated green synthesis of silver nanoparticles and their antimicrobial potential. Artificial cells, nanomedicine, and biotechnology, 44(6): 15691575.

Singh, S., Bhatia, R., Singh, A., Singh, P., Kaur, R., Khare, P., ... and Bhadada, S.K. 2018. Probiotic attributes and prevention of LPS-induced pro-inflammatory stress in RAW264. 7 macrophages and human intestinal epithelial cell line (Caco-2) by newly isolated Weissella cibaria strains. Food $\mathcal{E}$ function, 9(2): 1254-1264.
Yu, H.S., Lee, N.K., Choi, A.J., Choe, J.S., Bae, C.H. and Paik, H.D. 2019. Anti-inflammatory potential of probiotic strain Weissella cibaria JW15 isolated from Kimchi through regulation of NF- $\kappa \mathrm{B}$ and MAPKs pathways in LPS-induced RAW 264. 7 Cells. Journal of Microbial Biotechnology, 29(7): 1022-32.

Zannini, E., Mauch, A., Galle, S., Gänzle, M., Coffey, A., Arendt, E.K. and Waters, D.M. 2013. Barley malt wort fermentation by exopolysaccharide-forming Weissella cibaria MG 1 for the production of a novel beverage. Journal of Applied Microbiology, 115(6). 
